\title{
High-Sensitive Cardiac Troponin T for Prediction of Cardiovascular Outcomes in Stable Maintenance Hemodialysis Patients: A 3-Year Prospective Study
}

\author{
Linlin Sun Yonglan Wang Nan Zhang Xinmiao Xie Miao Ding Hui Guo \\ Fengqin Li Xiaoxia Wang
}

Department of Nephrology, Tongren Hospital, Shanghai Jiao Tong University School of Medicine, Shanghai, China

\author{
Keywords \\ Prognosis · High-sensitive cardiac troponin $\mathrm{T}$. \\ Echocardiography · Cardiovascular events - End-stage renal \\ disease $\cdot$ Hemodialysis
}

\begin{abstract}
Background: Hemodialysis patients, who are often excluded from cardiovascular (CV) clinical trials, are associated with higher CV morbidity and mortality. The risk stratification scheme for these patients is lacking. Therefore, this investigation examined the independent CV prognostic value of high-sensitive cardiac troponin T (hs-cTnT) and added prognostic value over echocardiographic parameters and other clinical risk predictors in asymptomatic stable maintenance hemodialysis (MHD) patients. Methods: 181 patients with end-stage renal disease undergoing MHD were eligible from the dialysis center of Tongren Hospital, Shanghai Jiao Tong University School of Medicine between October 2017 and September 2018. These patients were followed until September 2020 or until death. The median follow-up was 31 (IQR: 21-33) months. Outcome measures were all-cause mortality, first fatal or nonfatal CV events (CVEs), and 4-point composite major adverse CVEs (MACE). We performed multivariable Cox regression analysis using demographic, clinical, laboratory, and echocardiographic data to identify predictors of CV outcomes. We also evaluated the increased dis-
\end{abstract}

criminative value associated with the addition of echocardiographic parameters and hs-cTnT using net reclassification improvement (NRI) and integrated discrimination improvement (IDI). Results: During follow-up, 37 patients died, 84 patients suffered one or more CVEs, and 78 patients developed 4-point MACE. In univariable analyses, age, dialysis vintage, diastolic blood pressure, parathyroid hormone concentrations, hs-cTnT, B-type natriuretic peptide, left ventricular mass index (LVMI), and $E / E^{\prime}$ predicted all end points. hs-cTnT remained a strong predictor for each end point in multivariate analysis, whereas LVMI and $E / E^{\prime}$ did not. The addition of hs-cTnT on top of clinical and echocardiographic variables was associated with improvements in reclassification for CVEs (NRI = 44.6\% [15.9-74.3\%], IDI = 15.9\% [5.7$31.0 \%$, all $p<0.001)$, all-cause mortality (NRI = 35.5\% [10.1$50.2 \%], p<0.001, \mathrm{IDI}=4.4 \%[1.3-8.5 \%], p=0.005)$, and 4-point MACE (NRI = 47.2\% [16.1-64.9\%], $p<0.001$, IDI = $16.9 \%$ [5.5-37.3\%], $p=0.005$ ). Adding echocardiographic variables on top of clinical variables and hs-cTnT was not associated with significant improvements in NRI and IDI (all $p>0.05$ ). Conclusions: Our data suggest that hs-cTnT is a powerful independent predictor of CV outcome and allcause mortality in stable MHD patients. The additional use of echocardiography for improvement of risk stratification is not supported by our results.

(C) 2021 The Author(s) Published by S. Karger AG, Basel karger@karger.com www.karger.com/kbr

Karger $\stackrel{\text { ' }}{5}$

GOPEN ACCESS
(C) 2021 The Author(s)

Published by S. Karger AG, Basel

This is an Open Access article licensed under the Creative Commons Attribution-NonCommercial-4.0 International License (CC BY-NC) (http://www.karger.com/Services/OpenAccessLicense), applicable to the online version of the article only. Usage and distribution for commercial purposes requires written permission.
Correspondence to:

Xiaoxia Wang, omaha198501@163.com 


\section{Introduction}

Patients with end-stage renal disease (ESRD) are at a high risk of developing cardiovascular (CV) disease [1]. The CV mortality risk in patients with ESRD starting dialysis was estimated to be 15 -fold higher compared with the age-, race-, and sex-matched general population [2]. Despite the high prevalence of CV disease, patients with ESRD are often excluded from CV clinical trials and risk stratification scheme is lacking. Hence, better risk stratification is of considerable interest for targeted implementation of preventive therapies in ESRD patients.

A number of the previous studies have indicated that some biochemical markers, such as cardiac troponin $\mathrm{T}$ (cTnT), B-type natriuretic peptide (BNP), and some echocardiographic parameters, predict $\mathrm{CV}$ outcomes and mortality in ESRD patients, but the majority were retrospective, only measured 1 or 2 biomarkers, or used traditional assays for plasma biomarker testing [3-9]. Recently, as the high-sensitivity assay has been introduced, high-sensitive cTnT (hs-cTnT), with high sensitivity and specificity for myocardial injury, has been recommended as the primary basis of the risk stratification for acute coronary syndrome [10]. Using the highly sensitive assay, a moderately high prevalence of detectable hs-cTnT has been found in the general population and has been associated with an increased mortality risk [11]. An elevation of hs-cTnT has also been frequently detected in a proportion of chronic hemodialysis patients without evidence of acute myocardial ischemia [12-14], and the clinical significance as well as the prognostic value has remained controversial [5, 15-19].

Moreover, in line with literatures, the elevation of hs$\mathrm{cTnT}$ in our recent study in chronic hemodialysis patients showed strong correlation with left ventricular diastolic dysfunction, another CV outcome predictor, detected by tissue Doppler imaging (TDI) [12,13]. Therefore, the question as to whether hs-cTnT independently predicted CV outcomes after adjusting for important potential confounders, such as natriuretic peptides, cardiac hypertrophy, and dysfunction, remains unanswered. In the present study, we aimed to investigate whether the longterm CV prognostic value of hs-cTnT remained independent of other CV outcome predictors and potential confounders for hs-cTnT in a 3-year follow-up study involving 181 asymptomatic Chinese stable maintenance hemodialysis (MHD) patients.

cTnT to Predict CV Outcomes in

Hemodialysis Patients

\section{Materials and Methods}

\section{Patients}

This prospective observational cohort study was performed in accordance with the second Helsinki Declaration and approved by the Ethics Committee of Tongren Hospital Affiliated to Shanghai Jiao tong University. All included participants provided written informed consent.

Patients were eligible for study inclusion if they had ESRD, were on continuous ambulatory dialysis therapy for over 3 months, and did at least 3 4-h long hemodialysis sessions per week. Exclusion criteria included age $<18$ years, NYHA class III or IV heart failure, significant valvular disease, myocardial infarction within the last 6 months, active angina, underlying malignancy, current treatment for infection, major surgery within one month, and refusal to give consent.

Of the 212 MHD patients in our center, 31 (15\%) were excluded, and we included 181 patients on chronic hemodialysis between October 2017 and September 2018. All of them were followed until September 2020 or until death. Patients were censored for further follow-up if they underwent kidney transplantation or changed dialysis modality. The flowchart of the study is presented in Figure 1.

\section{Candidate Predictors}

Candidate predictors were selected based on clinical relevance and literature, including patient demographic details, comorbidities, anemia, volume status, mineral metabolism, Kt/V, cholesterol, hs-cTnT, BNP, and echocardiographic parameters. At baseline, patient demographics including gender, age, height, weight, body mass index (BMI), dialysis vintage, primary kidney disease, and comorbidities were obtained from medical records and from the patients themselves using a case-report form. The evaluation included the patients' medical records for cardiac factors and history of cardiac disease and assessment of the hydration status. Routine measurements of systolic and diastolic blood pressure were performed by dialysis nurses using a standardized mercury sphygmomanometer with the patient sitting, directly prior to dialysis, on the same day of the echocardiogram. The mean of 2 measurements was used for data analysis.

At study entry, blood was collected immediately before the 2nd or 3rd dialysis session of the week for measurements of hs-cTnT and $\mathrm{BNP}$ on the same day of the echocardiogram. Assays for hs-cTnT and BNP were performed at the central laboratory of Tongren hospital. Laboratory personnel were blinded to patients' outcome data or history at the time of assay. We used routine monthly laboratory work for values of serum hemoglobin, calcium, phosphorus, parathyroid hormone, albumin, cholesterol, and Kt/V. The results closest to the time of the echocardiogram were used.

Echocardiograms were performed immediately before dialysis using a SIMENS ACUSON Sequoia Model 512 with a $3.5 \mathrm{MHz}$ transducer by an experienced cardiologist blinded to patient clinical characteristics and outcomes. Two-dimensional measurements and TDI were performed in all patients as recommended by the American Society of Echocardiography. Measurements were averaged over 3 cardiac cycles. Left ventricular mass index (LVMI), left atrial diameter, left ventricular ejection fraction, and peak early $(E)$ and late $(A)$ transmitral flow velocities were all recorded. The early peak diastolic annular velocity $\left(\mathrm{E}^{\prime}\right)$ was measured by TDI from the septal mitral annulus, and the ratio of $E / E^{\prime}$ was calculated.

Kidney Blood Press Res 2021;46:484-494 


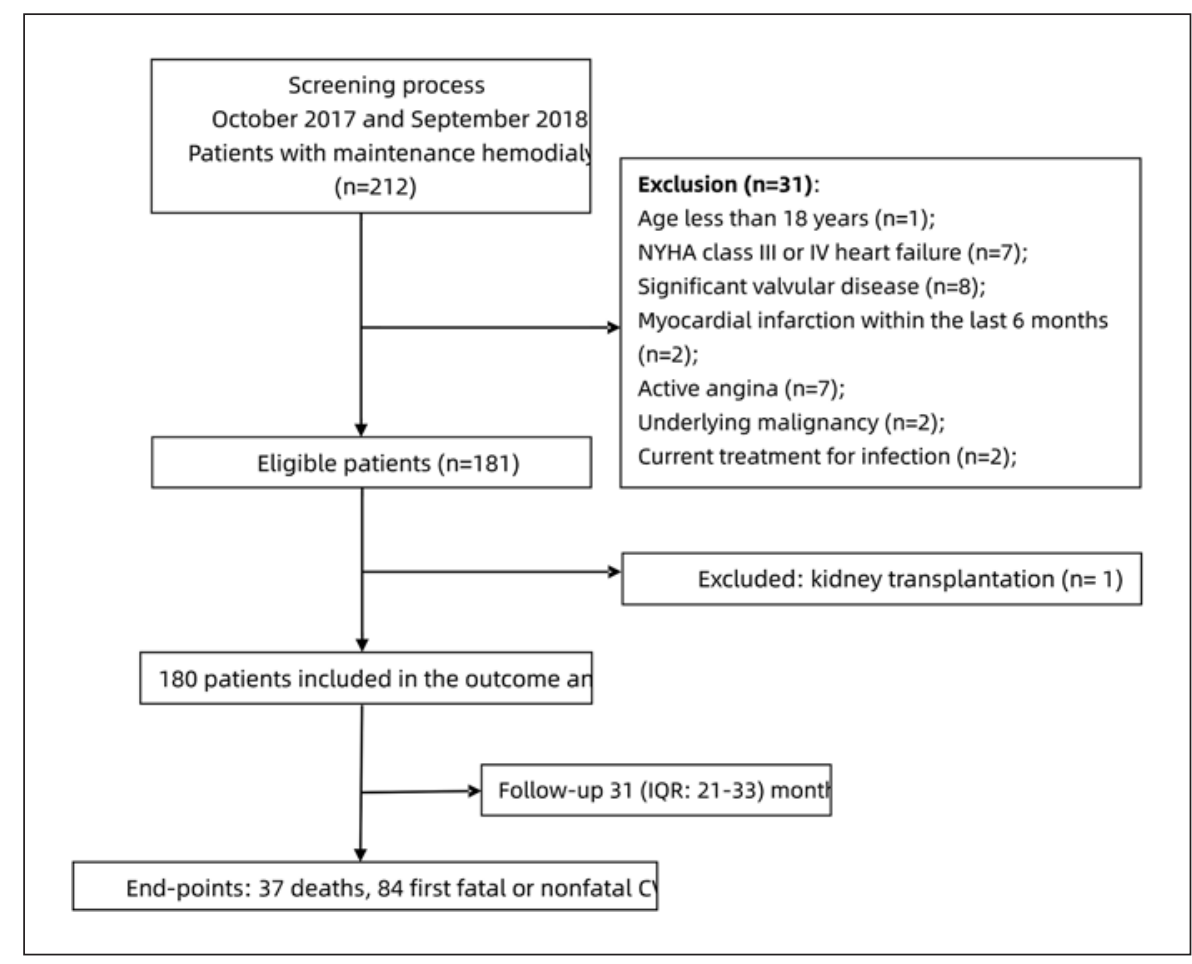

Fig. 1. Flowchart of the study.

\section{Outcomes}

All patients were followed up prospectively for 31 (IQR: 21-33) months from the day of study entry (when they had all of the baseline assessments) until September 2020 for CV outcomes. No patient was lost to follow-up. Cause of death and nature of the first CVE (fatal or nonfatal) were determined by the attending physicians, who were blinded to the baseline laboratory data and echocardiographic results. In case of death out of hospital, family members were interviewed by telephone to ascertain the circumstances surrounding death.

The outcome analyzed was first CVE (fatal or nonfatal), allcause mortality, or 4-point composite major adverse CVEs (MACE). Fatal and nonfatal CVEs included angina with electrocardiographically documented changes of myocardial ischemia, myocardial infarction, electrocardiographically documented arrhythmia, transient ischemic attacks, thromboembolic or hemorrhagic stroke (all defined according to conventional clinical criteria), congestive heart failure, and sudden cardiac death. Arrhythmia included atrial fibrillation, atrial flutter, atrial tachycardia, ventricular tachycardia, ventricular fibrillation, and II- or III-degree atrioventricular block. Congestive heart failure was defined clinically by the presence of symptoms and signs of heart failure including dyspnea, increased jugular venous pressure, and radiographic evidence of pulmonary venous congestion or interstitial edema. Sudden cardiac death was defined as unexpected natural death within $1 \mathrm{~h}$ from symptom onset and without any prior condition that would appear fatal. For patients who had multiple CVEs, survival analysis in relation to CVE was limited to the first CVE. The definition of 4-point MACE was composite of nonfatal myocardial infarction, nonfatal stroke, congestive heart failure, and CV mortality. Cardiac mortality was defined as any death with a demonstrable CV cause or sudden cardiac death.

\section{Statistical Analysis}

There were no missings in our 181 studied patient data. Continuous data were expressed as mean \pm SD or median (interquartile ranges) if distribution was skewed and compared using a student's $t$ test or rank-sum test. Categorical variables were presented as a percentage of participants and compared using a $\chi^{2}$ test. Plasma hs-cTnT and BNP concentrations were log transformed. The association of plasma hs-cTnT concentrations with other baseline variables was analyzed by Spearman's rank correlation.

We calculated survival probabilities using the Kaplan-Meier method plotted in survival graphs and compared using the logrank test. In this analysis, patients who underwent kidney transplantation or transferred to peritoneal dialysis during the 3-year period were censored at the time of transfer to alternative renal replacement therapy.

Univariable and multivariable Cox proportional hazard models were used to assess the associations between predictors and outcome rates. For the final multivariable cox model, we attempted to not cross the 10 events/nonevents per predictor variable criterion [20]. Therefore, we first selected the predictors based on clinical relevance, literature, and the baseline descriptive. $p<0.05$ was used to select a predictor after univariable screening. In case there was multicollinearity between variables, the variable with the best predictive value (i.e., combination of $p$ value $<0.05$ and type of predictor variable) was selected. The most relevant predictors after univariable screening and some traditional CV risk factors were used in the multivariable Cox regression model, and a backward stepwise procedure was used to choose the final model, with variables significant at $p<0.1$ being retained in the model. To clarify the effects of hs-cTnT and other variables, using the same variable selection procedure, with hs-cTnT, LVMI, or $E / E^{\prime}$ as exposure variables, different models were tested: model 1 included 
age, gender, and dialysis vintage; model 2 included age, gender, dialysis vintage, diastolic blood pressure, parathyroid hormone concentrations, history of left ventricular hypertrophy (LVH), history of ischemic heart disease (IHD), and diabetes mellitus; model 3 included variables from model 2 and BNP; and model 4 included variables from model 3 and hs-cTnT (for all analyses with echocardiographic parameters as exposure variables) or LV function (for analyses with hs-cTnT as exposure variable).

As recommended by the TRIPOD statement [21], the increased discriminative value associated with the addition of hs-

Table 1. Patient characteristics

$\begin{array}{lc}\text { Age, years } & 63.4 \pm 11.2 \\ \text { Female gender, } n(\%) & 79(43.6) \\ \text { BMI, kg/m }{ }^{2} & 22.4 \pm 2.8 \\ \text { Dialysis vintage, months } & 24.8(16.7-41.5) \\ \text { Smoking, } n(\%) & 51(28.2) \\ \text { History of LVH, } n(\%) & 146(80.7) \\ \text { History of IHD, } n(\%) & 91(50.3) \\ \text { Diabetes, } n(\%) & 87(48.1) \\ \text { Hypertension, } n(\%) & 172(95) \\ \text { SBP, mm Hg } & 147.5 \pm 12.4 \\ \text { DBP, mm Hg } & 90.1 \pm 8.3 \\ \text { Kt/V } & 1.65(1.43-1.91) \\ \text { Scr, } \mu \text { mol/L } & 963.0 \pm 175.3 \\ \text { Albumin, g/L } & 39.5(34.9,41.2) \\ \text { Hemoglobin, g/L } & 110 \pm 18.7 \\ \text { Cholesterol, mmol/L } & 5.3 \pm 1.0 \\ \text { Calcium, mmol/L } & 2.1 \pm 0.1 \\ \text { Phosphorus, mmol/L } & 2.0 \pm 0.3 \\ \text { iPTH, pg/mL } & 345(178-643) \\ \text { hs-cTnT, pg/mL } & 44.9(28.1-72.9) \\ \text { BNP, pg/mL } & 422(231-669) \\ \text { Anuric patients } & \\ \quad \text { (urine } 24-h \text { volume }<100 \mathrm{~mL}), n(\%) & 147(81.2) \\ \text { Ultrafiltration volume }(\mathrm{kg} / \mathrm{each} \text { time) } & 2.5 \pm 1.7 \\ \text { LVMI, g/m }{ }^{2} & 139.4 \pm 14.1 \\ \text { LVEF, \% } & 60.1 \pm 6.6 \\ \text { E/E' } & 12.1 \pm 4.2 \\ \text { LAD, mm } & 34.9 \pm 4.7 \\ \text { E/A } & 1.1 \pm 0.6 \\ \end{array}$

BMI, Body mass index; LVH, left ventricular hypertrophy; IHD, ischemic heart disease; SBP, systolic blood pressure; DBP, diastolic blood pressure; hs-cTnT, high-sensitive cardiac tropnin T; BNP, B-type natriuretic peptide; Scr, serum creatinine; iPTH, intact parathyroid hormone; LVMI, left ventricular mass index; $L V E F$, left ventricular ejection fraction; $E / E^{\prime}$, diastolic left ventricular function; LAD, left atrial diameter.

Fig. 3. Kaplan-Meier survival curve analysis with subsequent logrank test (end points: first fatal or nonfatal CVE, all-cause mortality, and 4-point MACE). Event-free survival in patients with MHD stratified by tertiles of hs-cTnT $(\mathbf{A}, \mathbf{D}, \mathbf{G})$, tertiles of LVMI (B, E, H), and tertiles of diastolic left ventricular function $\left(E / E^{\prime}\right)(\mathbf{C}, \mathbf{F}, \mathbf{I}) . \mathrm{CV}$,

cTnT to Predict CV Outcomes in

Hemodialysis Patients
cTnT or cardiographic variables on top of the aforementioned covariates was evaluated using net reclassification improvement (NRI) and integrated discrimination improvement (IDI) [22-24]. SPSS19.0 statistical software and the R software were applied to this analysis.

\section{Results}

\section{Baseline Characteristics and Outcomes}

The baseline characteristics of the patient population are shown in Table 1. Briefly, the patients studied comprised 102 men and 79 women with a mean age of $63.4 \pm$ 11.24 years; BMI was $22.4 \pm 2.83 \mathrm{~kg} / \mathrm{m}^{2}$. The median dialysis vintage was 24.8 (16.7-41.5) months. Diabetes was present in 87 (48.1\%) of our participants, hypertension in 172 (95\%), history of IHD in 91 (50.3\%), and history of LVH in $146(80.7 \%)$. Causes of renal failure in patients included chronic glomerulonephritis $(n=69)$, diabetic nephropathy $(n=52)$, hypertensive nephrosclerosis $(n=$

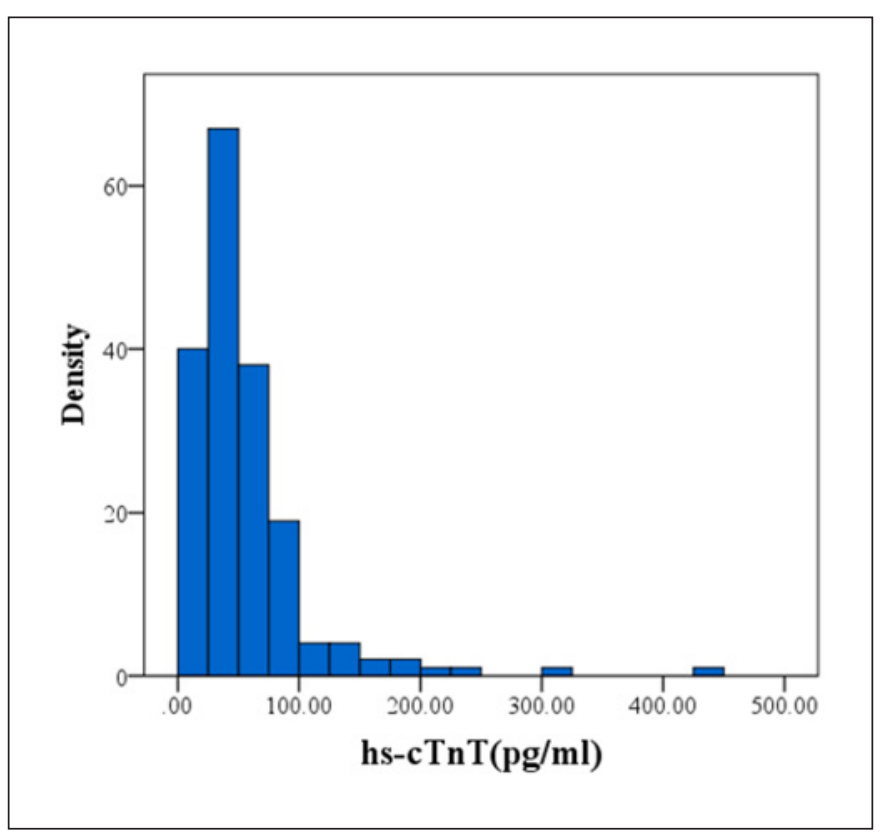

Fig. 2. Distribution of hs-cTnT concentrations. hs-cTnT, highsensitive cardiac troponin $\mathrm{T}$.

cardiovascular; CVE, CV event; MACE, major adverse CV events; MHD, maintenance hemodialysis; hs-cTnT, high-sensitive cardiac troponin T; LVMI, left ventricular mass index; $E / E^{\prime}$, diastolic left ventricular function.

(For figure see next page.) 
A

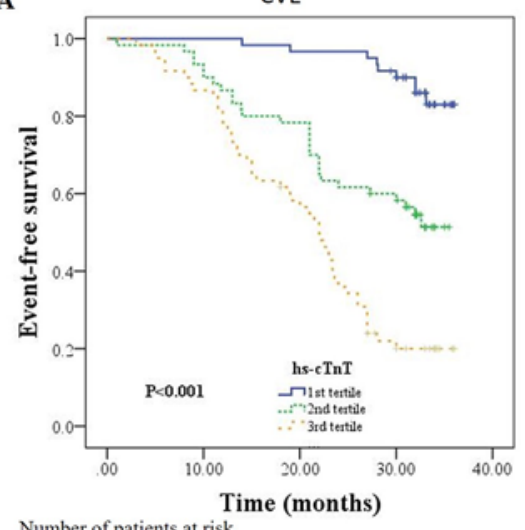

Number of patients at risk

$\begin{array}{ccccc}1^{\text {st }} \text { tertile } & 60 & 60 & 58 & 29 \\ 2^{\text {nd }} \text { tertile } & 60 & 56 & 47 & 35 \\ 3^{\text {rd }} \text { tertile } & 60 & 52 & 33 & 6\end{array}$

D

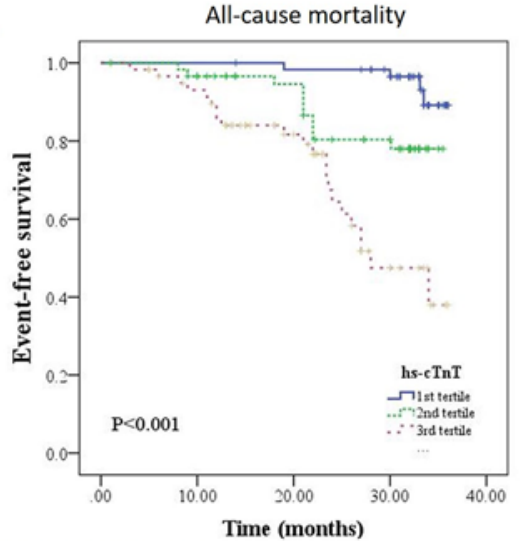

Number of patients at risk

$\begin{array}{lllll}1^{\text {It }} \text { tertile } & 60 & 60 & 56 & 29\end{array}$

$2^{\text {nd }}$ tertile $59 \quad 52 \quad 45 \quad 18$

$\begin{array}{lllll}3^{\text {rd }} \text { tertile } & 58 & 46 & 28 & 6\end{array}$

G

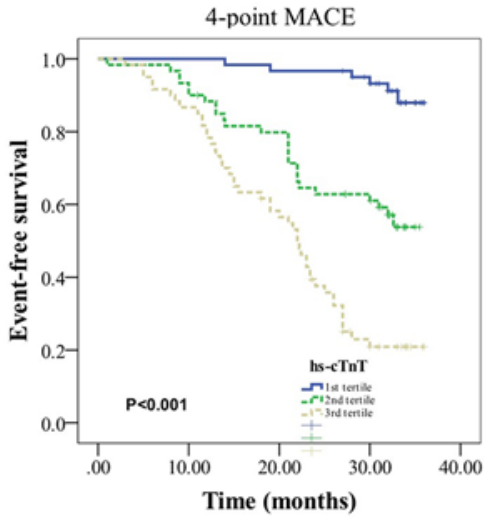

Number of patients at risk

$\begin{array}{lllll}1^{\text {st }} \text { tertile } & 60 & 60 & 40 & 18 \\ 2^{\text {nd }} \text { tertile } & 60 & 56 & 47 & 35 \\ 3^{\text {rd }} \text { tertile } & 60 & 52 & 34 & 11\end{array}$

B

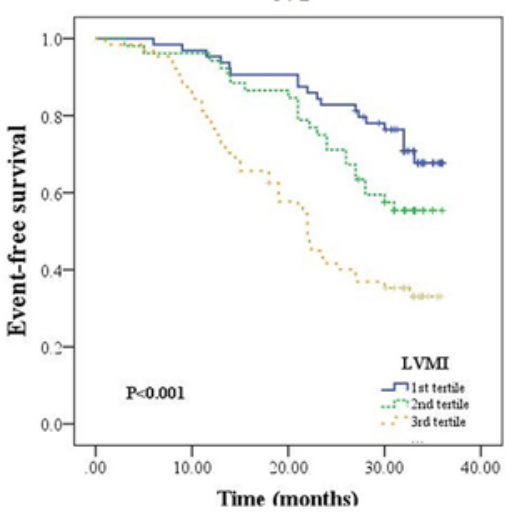

Number of patients at risk

$\begin{array}{lllll}1^{\text {st }} \text { tertile } & 64 & 62 & 57 & 26 \\ 2^{\text {nd }} \text { tertile } & 52 & 50 & 45 & 16 \\ 3^{\text {sd }} \text { tertile } & 64 & 56 & 36 & 13\end{array}$

E

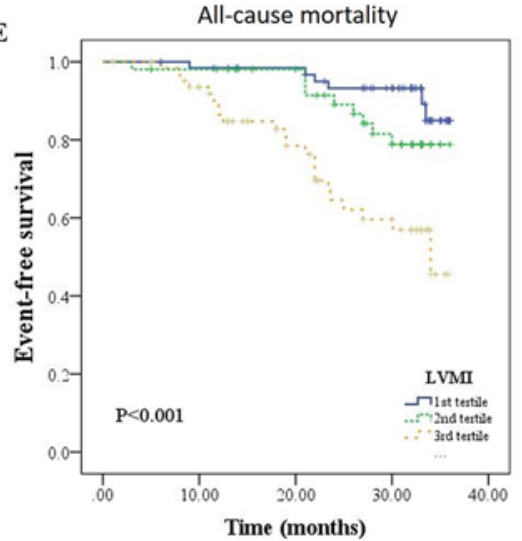

Number of patients at risk

$1^{\text {sh }}$ tertile $60 \quad 60$

$2^{\text {sat }}$ tertile 59

$3^{\text {sd }}$ tertile 58

H

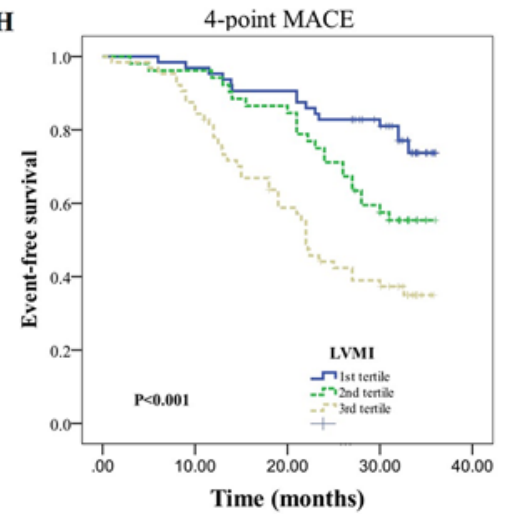

Number of patients at risk

$\begin{array}{lllll}1^{\text {st }} \text { tertile } & 64 & 62 & 55 & 26 \\ 2^{\text {nd }} \text { tertile } & 52 & 50 & 45 & 30 \\ 3^{\text {rd }} \text { tertile } & 64 & 56 & 36 & 23\end{array}$

C

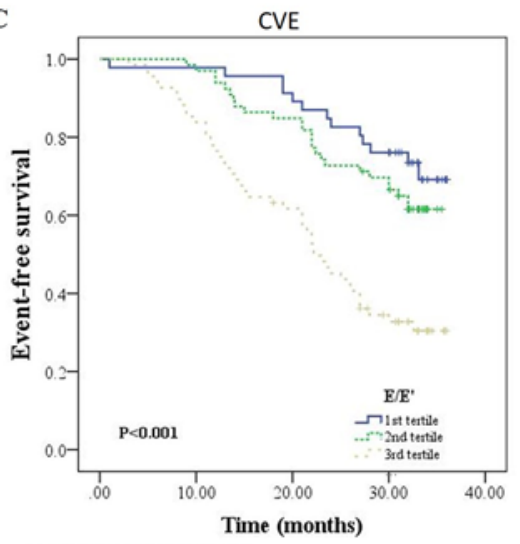

Number of patients at risk

$\begin{array}{lllll}1^{\text {st }} \text { tertile } & 46 & 45 & 42 & 19 \\ 2^{\text {nd }} \text { tertile } & 66 & 65 & 56 & 25 \\ 3^{\text {rd }} \text { tertile } & 68 & 58 & 40 & 11\end{array}$

F

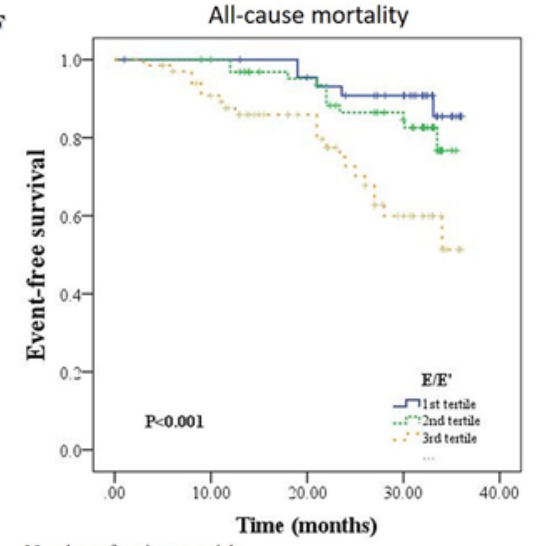

Number of patients at risk

$1^{\text {st }}$ tertile $46 \quad 45 \quad 40 \quad 18$

$2^{\text {nd }}$ tertile $66 \quad 62 \quad 53 \quad 24$

$3^{\text {rd }}$ tertile $66 \quad 51 \quad 36 \quad 11$

I

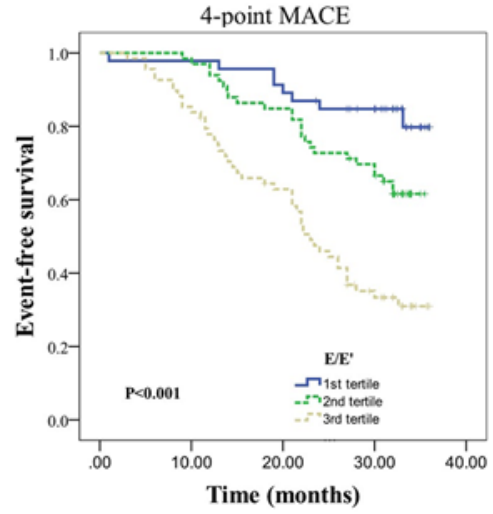

Number of patients at risk

$\begin{array}{lllll}1^{\text {st }} \text { tertile } & 46 & 45 & 42 & 35\end{array}$

$2^{\text {nd }}$ tertile $66 \quad 65 \quad 56 \quad 45$

$3^{\text {rd }}$ tertile $68 \quad 58 \quad 41 \quad 20$ 
Table 2. Univariable Cox proportional regression analyses for all-cause mortality, first fatal or nonfatal CVE, and 4-point MACE

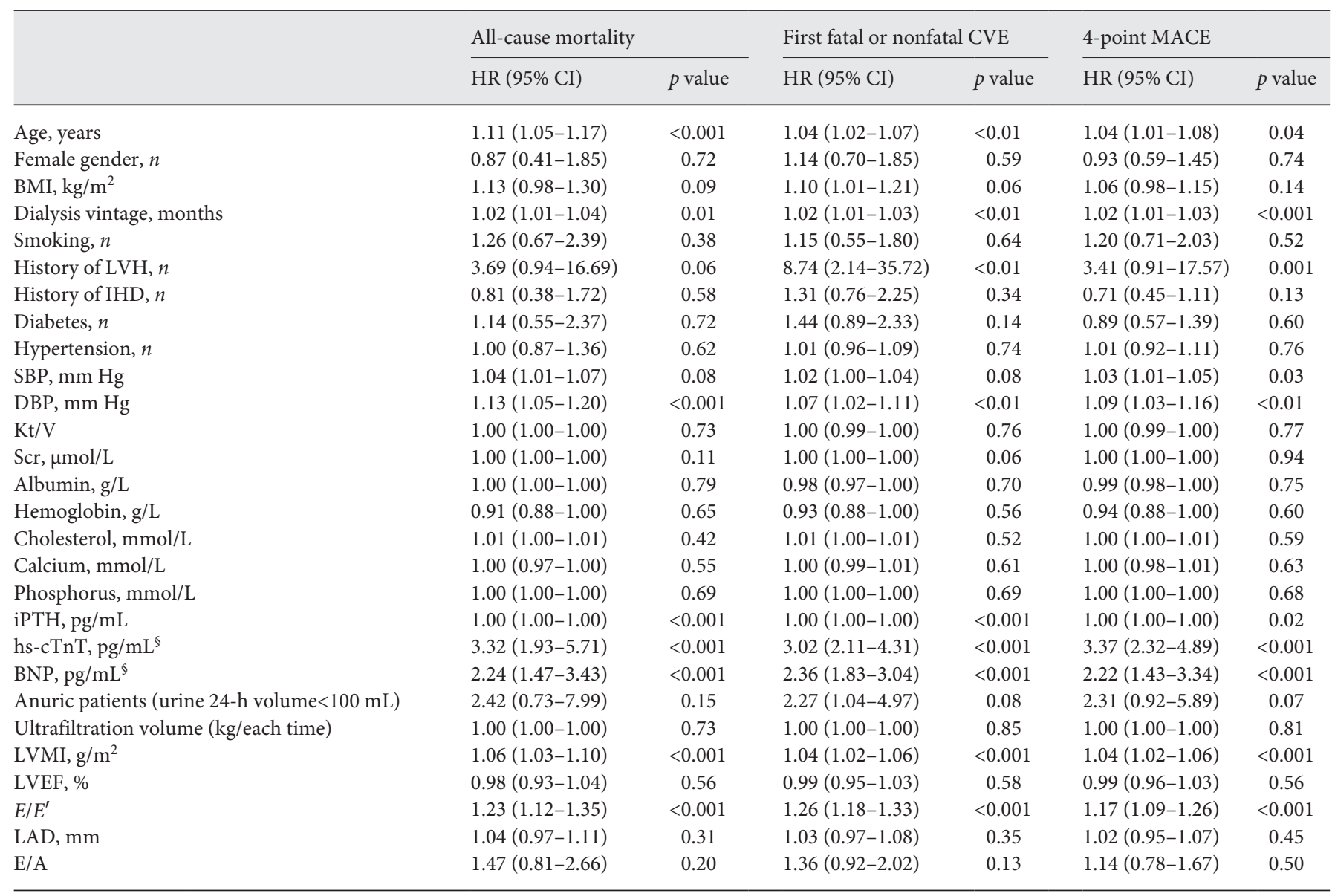

4-point MACE: composite of nonfatal myocardial infarction, nonfatal stroke, congestive heart failure, and CV mortality. BMI, Body mass index; LVH, left ventricular hypertrophy; IHD, ischemic heart disease; SBP, systolic blood pressure; DBP, diastolic blood pressure; hs-cTnT, high-sensitive cardiac tropnin T; BNP, B-type natriuretic peptide; Scr, serum creatinine; iPTH, intact parathyroid hormone; LVMI, left ventricular mass index; LVEF, left ventricular ejection fraction; HR, hazard ratio; CI, confidence interval; CV, cardiovascular; CVE, CV event; MACE, major adverse CV events; LAD, left atrial diameter. ${ }^{\S} \ln$ transformed before analysis.

37), polycystic kidney disease $(n=6)$, chronic interstitial nephritis $(n=5)$, lupus nephritis $(n=3)$, and other not identified causes $(n=9)$. The distribution of hs-cTnT was shown in Figure 2. The median value of hs-cTnT was 44.2 (26.2-64.3) $\mathrm{pg} / \mathrm{mL}$. All participants had detectable hscTnT and hs-cTnT concentrations exceeded the diagnostic cutoff of $14 \mathrm{pg} / \mathrm{mL}$ in 167 (93\%) patients.

During follow-up, 37 (20.4\%) patients died, and 1 patient underwent kidney transplantation (Fig. 1). The median follow-up was 31 (IQR: 21-33) months. There were 29 deaths from CV causes, including IHD/myocardial infarction in 9, congestive heart failure in 4, arrhythmia in 2 , sudden death in 6 , and cerebrovascular disease in 8 patients. The remaining 8 non-CV deaths were due to infections in 3 , malignancy in 3 , and non-CV hemorrhage in
2 patients. Among the study subjects, 84 (46.4\%) patients developed one or more fatal or nonfatal CVEs: 21 patients had IHD, 22 patients had cerebrovascular disease, 32 patients had congestive heart failure, 3 patients had arrhythmia, and 6 patients had sudden cardiac death as their first CVE. During follow-up, 78 patients developed 4-point MACE including nonfatal myocardial infarction in 10, nonfatal stroke in 11, nonfatal congestive heart failure in 28 , and CV mortality in 29 patients.

\section{Correlations between hs-cTnT and Other Baseline Variables}

According to univariate correlation analysis (see online suppl. Table 1; for all online suppl. material, see www.karger.com/doi/10.1159/000516658), serum hs- 
Table 3. Multivariate Cox proportional regression analyses for first fatal or nonfatal CVE, all-cause mortality, and 4-point MACE

\begin{tabular}{|c|c|c|c|c|c|c|c|c|}
\hline hs-cTnT, pg/mL $L^{\S}$ & $3.29(2.26-4.79)$ & $<0.001$ & $5.03(3.13-8.08)$ & $<0.001$ & $4.39(2.73-7.08)$ & $<0.001$ & $3.93(2.19-7.03)$ & $<0.001$ \\
\hline LVMI, $g / \mathrm{m}^{2}$ & $1.03(1.01-1.05)$ & $<0.01$ & $1.02(1.00-1.04)$ & 0.02 & $1.02(1.00-1.04)$ & 0.04 & $1.00(0.98-1.02)$ & 0.93 \\
\hline$E / E^{\prime}$ & $1.14(1.07-1.21)$ & $<0.001$ & $1.15(1.07-1.24)$ & $<0.001$ & $1.14(1.06-1.23)$ & $<0.001$ & $1.03(0.95-1.12)$ & 0.41 \\
\hline hs-cTnT, pg/mL $L^{\S}$ & $3.31(1.86-5.89)$ & $<0.001$ & $4.96(2.32-10.58)$ & $<0.001$ & $4.01(1.89-8.50)$ & $<0.001$ & $3.08(1.20-7.92)$ & 0.02 \\
\hline LVMI, $g / \mathrm{m}^{2}$ & $1.05(1.02-1.07)$ & $<0.001$ & $1.04(1.01-1.06)$ & $<0.01$ & $1.03(1.00-1.06)$ & 0.02 & $1.01(0.98-1.04)$ & 0.43 \\
\hline$E / E^{\prime}$ & $1.14(1.04-1.24)$ & $<0.01$ & $1.14(1.02-1.27)$ & 0.02 & $1.12(1.00-1.25)$ & 0.05 & $1.04(0.92-1.18)$ & 0.56 \\
\hline \multicolumn{9}{|l|}{ 4-point MACE } \\
\hline hs-cTnT, pg/mL $L^{\S}$ & $3.81(2.58-5.64)$ & $<0.001$ & $5.58(3.41-9.12)$ & $<0.001$ & $4.88(2.96-8.04)$ & $<0.001$ & $3.75(2.05-6.89)$ & $<0.001$ \\
\hline LVMI, $g / \mathrm{m}^{2}$ & $1.04(1.02-1.06)$ & $<0.001$ & $1.03(1.01-1.05)$ & $<0.01$ & $1.03(1.01-1.05)$ & $<0.01$ & $1.01(0.99-1.03)$ & 0.36 \\
\hline
\end{tabular}

Model 1 is adjusted for age, gender, and dialysis vintage. Model 2 is adjusted for age, gender, dialysis vintage, diastolic blood pressure, parathyroid hormone concentrations, prevalent LVH, IHD, and DM. Model 3 includes variables from model 2 and BNP. Model 4 includes variables from model 3 and hscTnT (for all analyses with echocardiographic parameters as exposure variables) or LV function (for analyses with hs-cTnT as exposure variable). 4-point MACE: composite of nonfatal myocardial infarction, nonfatal stroke, congestive heart failure, and CV mortality. HR, hazard ratio; 95\% CI, 95\% confidence interval; hs-cTnT, high-sensitive cardiac tropnin T; BNP, B-type natriuretic peptide; LVMI, left ventricular mass index; E/E', diastolic left ventricular function; CV, cardiovascular; CVE, CV event; MACE, major adverse CV events. ${ }^{\mathbb{S}} \ln$ transformed before analysis.

cTnT showed a significantly positive correlation with higher BMI $(r=0.34, p<0.001)$, longer dialysis vintage $(r=0.40, p<0.001)$, higher blood pressure $(r=0.48$, $p<0.001)$, higher serum creatinine $(r=0.29, p<0.001)$, higher parathyroid hormone $(r=0.41, p<0.001)$, higher BNP $(r=0.35, p<0.001)$, higher LVMI $(r=0.59$, $p<0.001)$, and $E / E^{\prime}$ value $(r=0.68, p<0.001)$. For better understanding of the relationships, scatterplots of the correlation between hs-cTnT and LVMI, hs-cTnT and $E / E^{\prime}$, and LVMI and $E / E^{\prime}$ are provided as online suppl. Figure 1 .

\section{Survival Analysis}

Figure 3 shows the Kaplan-Meier survival curves in relation to first fatal or nonfatal CVE, all-cause mortality, and 4-point MACE of patients in the tertile groups of hscTnT (1st tertile group: hs-cTnT <33.1 pg/mL, 2nd tertile group: hs-cTnT $33.1 \sim 55.9 \mathrm{pg} / \mathrm{mL}$, and 3rd tertile group: hs-cTnT $>55.9 \mathrm{pg} / \mathrm{mL}$ ), LVMI (1st tertile group: LVMI $<136 \mathrm{~g} / \mathrm{m}^{2}, 2$ nd tertile group: LVMI $136 \sim 144 \mathrm{~g} / \mathrm{m}^{2}$, and 3rd tertile group: LVMI $>144 \mathrm{~g} / \mathrm{m}^{2}$ ), and $E / E^{\prime}$ (1st tertile group: $E / E^{\prime}<9.5$, 2nd tertile group: $E / E^{\prime} 9.5 \sim 15.2$, and 3 rd tertile group: $\left.E / E^{\prime}>15.2\right)$. Patients with the highest hscTnT, highest LVMI, and highest $E / E^{\prime}$ were all at highest risks for first fatal or nonfatal CVE, all-cause mortality, or 4-point MACE (hs-cTnT: log-rank $p<0.001$; LVMI: logrank $p<0.001$; and $\left.E / E^{\prime}: \log -\operatorname{rank} p<0.001\right)$.

\section{Univariable Analysis}

Univariable Cox proportional hazard regression analyses revealed that age, dialysis vintage, diastolic blood pressure, parathyroid hormone concentrations, hs-cTnT, BNP, LVMI, and $E / E^{\prime}$ were associated with a higher risk of first fatal or nonfatal CVE, all-cause mortality, and 4-point MACE (Table 2).

\section{Multivariable Analysis}

The final variables selected for inclusion in the model were age, gender, dialysis vintage, diastolic blood pressure, hs-cTnT, BNP, parathyroid hormone concentrations, history of IHD, history of LVH and DM, LVMI, and $E / E^{\prime}$. For the continuous predictors' age, dialysis vintage, hs-cTnT, BNP, parathyroid hormone concentrations, LVMI, and $E / E^{\prime}$, no multicollinearities were found using variance inflation factor.

In multivariate regression analyses (Table 3), hs-cTnT, LVMI, and $E / E^{\prime}$ remained predictors of adverse outcomes after adjustment for age, gender, and dialysis vintage (model 1) and additional adjustment for diastolic blood pressure, parathyroid hormone concentrations, history of LVH, IHD, diabetes, and BNP (model 3). Finally, hscTnT remained strongly and significantly associated with each end point after adjustment for LVMI and $E / E^{\prime}$. In contrast, LVMI and $E / E^{\prime}$ were no longer significant predictors of each end point after adjustment for hs-cTnT 
Table 4. Final stepwise multivariable Cox regression models for all-cause mortality, first fatal or nonfatal CVE, and 4-point MACE

\begin{tabular}{|c|c|c|c|c|c|c|}
\hline$N$ & 37 & & 84 & & 78 & \\
\hline Age, years & $1.05(1.01-1.09)$ & 0.01 & $1.02(1.00-1.04)$ & 0.08 & $1.04(1.01-1.09)$ & 0.07 \\
\hline History of IHD, $n$ & $0.34(0.15-0.73)$ & 0.01 & $0.31(0.16-0.82)$ & $<0.01$ & $0.28(0.14-0.79)$ & $<0.01$ \\
\hline Diabetes, $n$ & $1.98(0.92-4.27)$ & 0.08 & - & - & - & - \\
\hline hs-cTnT, $\mathrm{pg} / \mathrm{mL}^{\S}$ & $4.83(2.51-9.26)$ & $<0.001$ & $4.25(2.81-8.42)$ & $<0.001$ & $5.15(3.34-7.95)$ & $<0.001$ \\
\hline
\end{tabular}

Data are HR (95\% CI). 4-point MACE: composite of nonfatal myocardial infarction, nonfatal stroke, congestive heart failure, and CV mortality. IHD, ischemic heart disease; iPTH, intact parathyroid hormone; hs-cTnT, high-sensitive cardiac tropnin T; BNP, B-type natriuretic peptide; CVE, cardiovascular event; MACE, major adverse cardiovascular events; HR, hazard ratio; CI, confidence interval. $\$$ In transformed before analysis.

Table 5. NRI and IDI by adding the LVMI, $E / E^{\prime}$, and hs-cTnT to a model including covariables

\begin{tabular}{|c|c|c|c|c|}
\hline & NRI\% (95\% CI) & $p$ value & IDI\% (95\% CI) & $p$ value \\
\hline \multicolumn{5}{|c|}{ First fatal or nonfatal CVE } \\
\hline$E / E^{\prime a}$ & $0.9(0.1-8.5)$ & 0.59 & $0.1(-0.4$ to 1.0$)$ & 0.63 \\
\hline LVMI $^{\mathrm{a}}$ & $0.6(0.2-9.2)$ & 0.63 & $-0.2(-0.9$ to 1.3$)$ & 0.50 \\
\hline hs-cTnT ${ }^{b}$ & $44.6(15.9-74.3)$ & $<0.001$ & $15.9(5.7-31.0)$ & $<0.001$ \\
\hline \multicolumn{5}{|c|}{ All-cause mortality } \\
\hline$E / E^{\prime a}$ & $0.5(-0.3$ to 0.9$)$ & 0.67 & $-0.1(-1$ to 0.9$)$ & 0.58 \\
\hline LVMI $^{\mathrm{a}}$ & $1.6(0.4-2.7)$ & 0.64 & $0.7(0.3-1.7)$ & 0.34 \\
\hline hs-cTnT ${ }^{b}$ & $35.5(10.1-50.2)$ & $<0.001$ & $4.4(1.3-8.5)$ & 0.01 \\
\hline \multicolumn{5}{|c|}{ 4-point MACE } \\
\hline$E / E^{\prime a}$ & $0.8(0.1-8.1)$ & 0.57 & $0.2(-0.3$ to 1.4$)$ & 0.59 \\
\hline LVMI $^{\mathrm{a}}$ & $1.1(0.3-12.7)$ & 0.53 & $0.1(-0.5$ to 2.1$)$ & 0.63 \\
\hline hs-cTnT ${ }^{b}$ & $47.2(16.1-64.9)$ & $<0.001$ & $16.9(5.5-37.3)$ & $<0.001$ \\
\hline
\end{tabular}

NRI, net reclassification improvement; IDI, integrated discrimination improvement; CI, confidence interval, hs-cTnT, high-sensitive cardiac tropnin T, LVMI, left ventricular mass index, E/E', diastolic left ventricular function; CV, cardiovascular; CVE, CV event; MACE, major adverse CV events; BNP, B-type natriuretic peptide. 4-point MACE: composite of nonfatal myocardial infarction, nonfatal stroke, congestive heart failure, and CV mortality. ${ }^{a}$ The basic model includes covariables age, gender, dialysis vintage, history of LVH, IHD, and DM, diastolic blood pressure, parathyroid hormone concentrations, BNP, and hs-cTnT. ${ }^{b}$ The basic model includes covariables age, gender, dialysis vintage, history of LVH, IHD, and DM, diastolic blood pressure, parathyroid hormone concentrations, BNP, LVMI, and $E / E^{\prime}$.

(model 4). In the fully adjusted models (Table 4), hs-cTnT (first fatal or nonfatal CVE: HR 4.25 [2.81-8.42], $p<$ 0.001 ; all-cause mortality: HR 4.83 [2.51-9.26], $p<0.001$, 4-point MACE: HR 5.15 [3.34-7.95], $p<0.001$ ) was still found as a strongly independent risk factor for each end point, whereas LVMI and $E / E^{\prime}$ were not significant prognostic factors. In addition, we analyzed the subgroup of patients without history of IHD $(n=90)$ in the fully adjusted model and found that higher hs-cTnT was still an independent predictor for first fatal or nonfatal CVE (HR 5.26 [3.08-9.00], $p<0.001$ ), all-cause mortality (HR 8.88 [3.22-14.48], $p<0.001$ ), and 4-point MACE (HR 6.72 [3.64-10.46], $p<0.001)$.

We also evaluated the increased discriminative value associated with the addition of echocardiographic parameters and hs-cTnT using NRI and IDI (Table 5). The addition of hs-cTnT on top of the other risk factors and echocardiographic variables was associated with improve- 
ments in reclassification for first CVE (NRI $=44.6 \%$ [15.9-74.3\%], IDI $=15.9 \%$ [5.7-31.0\%], all $p<0.001)$, allcause mortality $(\mathrm{NRI}=35.5 \%[10.1-50.2 \%], p<0.001$, IDI $=4.4 \%[1.3-8.5 \%], p=0.005)$, and 4-point MACE $(\mathrm{NRI}=47.2 \%[16.1-64.9 \%], p<0.001$, IDI $=16.9 \%$ [5.5$37.3 \%$ ], $p=0.005)$. Adding echocardiographic variables on top of clinical variables and hs-cTnT was not associated with significant improvements in NRI and IDI (all $p>0.05)$.

\section{Discussion}

\section{Main Findings}

In this prospective study, using a highly sensitive assay for cTnT, we showed that a high serum level of hs-cTnT provided an important prognostic value for CVEs and allcause mortality in asymptomatic stable MHD patients independent of other risk predictors including background of IHD, diabetes, elevation of parathyroid hormone, BNP, cardiac hypertrophy, and dysfunction. In addition, our study demonstrated that the additional use of echocardiography for improvement of risk stratification is not supported by our results.

\section{Interpretation}

CTnT is a highly sensitive and specific marker of myocardial damage. It is useful in diagnosing acute myocardial infarction and predicting mortality in nonrenal failure patients with unstable coronary disease [25]. As the high-sensitivity assay was introduced in recent years, hscTnT may often be detected in chronic hemodialysis patients without evidence of acute myocardial ischemia. In our study, there were about $93 \%$ of patients with hs-cTnT levels above the 99th percentile upper reference limit (14 $\mathrm{pg} / \mathrm{mL}$ ) which was consistent with the literature reports $[12,13]$. Several studies have shown cTnT to be a strong predictor of cardiac events in hemodialysis patients [5, $15,16]$. However, some conflicting results have been reported. Bargnoux [17] suggested that BNP and C-reactive protein were independent predictors of mortality but not cardiac troponins. Satyan [18] reported in 150 HD patients without symptoms that NT-proBNP was strongly related with all-cause mortality and CV mortality, but troponin did not.

$\mathrm{LVH}$, as well as diastolic dysfunction, is established as a predictor of major adverse cardiac events in the general population and hemodialysis patients [26-30]. Since our analysis showed that the MHD patients with increased hs-cTnT had greater LV hypertrophy and worse diastolic function as reported in other studies $[12,13]$, we first investigated the prognostic value of echocardiographic parameters and confirmed that LVMI and $E / E^{\prime}$ predicted adverse CV outcomes and all-cause mortality in both univariable analyses and multivariable analyses after adjustment for conventional CV and uremia-related risk factors. These findings are in accordance with prior studies, but it is worth noting that these studies did not adjust for hs-cTnT.

Then, we confirmed hs-cTnT as a strong predictor of $\mathrm{CV}$ outcome and all-cause mortality in MHD patients. We found that hs-cTnT remained a strong outcome predictor even after full adjustment for potential confounders, particularly for echocardiographic parameters and BNP. However, LVMI and $E / E^{\prime}$ did not remain independent CV outcome predictors after adjustment for hscTnT. Higher LVMI and $E / E^{\prime}$ predicted neither CVEs nor all-cause mortality in the fully adjusted Cox model. These results are notable because our study demonstrated for the first time that although hs-cTnT was significantly correlated with LVMI and $E / E^{\prime}$ in stable MHD patients, its prognostic value was independent of these echocardiographic parameters. We consider one reason could be that increased hs-cTnT values among hemodialysis patients in the absence of clinically suspected ischemia may be not only the result of an increase of left ventricular filling pressure and wall tension but also the result of myocardial micronecrosis, subendocardial hypoperfusion, cell membrane permeability changes, and frequent changes of LV preload condition [31]. Another explanation might be that hypertension and $\mathrm{LVH}$ are prevalent in dialysis patients. The prevalence of hypertension and LVH in our patient population was 95 and $80.7 \%$, respectively. The third explanation might be that we excluded patients with NYHA class III or IV heart failure and severe IHD patients.

Furthermore, our current findings indicated that the addition of hs-cTnT on top of clinical and echocardiographic variables was associated with significant improvements in reclassification for CVEs and all-cause mortality in chronic MHD patients. However, adding echocardiographic variables on top of clinical variables and cardiac biomarkers was not associated with significant improvements in NRI and IDI. Based on our analysis, the additional use of echocardiography for improvement of risk stratification is not supported in patients with stable MHD.

The cTnT cutoffs, most studies using a traditional assay, used to evaluate mortality, and CV risk differed in different studies. By using a high-sensitive assay that can 
detect myocardial micronecrosis, the cutoff value of an increased hs-cTnT for predicting all-cause mortality, and CVEs is known little. There was some suggestion from our Kaplan-Meier analyses that the third group of hscTnT, an hs-cTnT $>55.9 \mathrm{pg} / \mathrm{mL}$, was associated with more adverse CV outcomes. Conversely, the first group of hs-cTnT, an hs-cTnT $<33.1 \mathrm{pg} / \mathrm{mL}$, may be useful in defining a subgroup of MHD patients with lower CV risk, as evident from our study.

\section{Limitations}

This study has several potential limitations. First, our study population comes from one single dialysis center, and our sample size is relatively small. Therefore, the generalizability of our findings remains to be determined. Second, a relatively large number of covariates were considered in the multivariable analysis for all-cause mortality despite the relatively small event numbers. Some of our results thus require interpretation with caution and need confirmation with larger prospective studies before being generally accepted. Third, since this study was limited to stable MHD patients without clinical heart symptoms, the associations between various biomarkers and cardiac outcomes may not be generalizable to other populations. Fourth, all biomarker measurements and echocardiography were performed only once during the patients' admission; therefore, it was difficult to demonstrate the impact of the changes in these biomarkers on patients' clinical outcomes. Future studies will be necessary to find out whether the changes in biomarkers over time have an association with the clinical outcomes. Fifth, the follow-up duration was short in this study. Despite this, these patients have continuously been followed up, and a better long-term study will be carried out in a near future. Sixth, cardiac biomarkers and echocardiographic parameters can be difficult to measure accurately among patients on hemodialysis due to frequent volume shifts. Although a blood sample was collected and the echocardiographic parameter was measured on the same day before dialysis, fluid status may affect the accuracy of the survival model.

\section{Conclusions}

In conclusion, our study suggests that hs-cTnT is a strong CV outcome and all-cause mortality predictor even after adjustment for potential confounders, particularly for $\mathrm{BNP}$ and echocardiographic parameters in stable MHD patients. The additional use of echocardiography for improvement of risk stratification is not supported by our results.

\section{Statement of Ethics}

The study was approved by the Ethics Committee of Tongren Hospital Affiliated to Shanghai Jiao Tong University (No. 2017051-01). All procedures performed in this study were in accordance with the ethical standards of the Institutional and International Research Committee and with the 1964 Helsinki Declaration and its later amendments or comparable ethical standards. All subjects have given their written informed consent.

\section{Conflict of Interest Statement}

The authors have no conflicts of interest to declare.

\section{Funding Sources}

This work was supported by the Natural Science Foundation of Shanghai Science and Technology Committee (20ZR1451600), the Science Project of Shanghai Municipal Health Bureau (201940439), the Science Project of Shanghai Municipal Health Bureau of Changning District (20174Y004), and the National Natural Science Foundation of China (81770718). The funding party does not have any role in the development and progress of the study nor in the publication process.

\section{Author Contributions}

Research idea and study design: L.L.S. and X.X.W.; data acquisition: Y.L.W., N.Z., X.X.M., M.D., H.G., and F.Q.L.; supervision or mentorship: X.X.W. and L.L.S. All the authors read and approved the final manuscript.

References

Kidney Blood Press Res 2021;46:484-494 DOI: $10.1159 / 000516658$ 2. e007876.
1 USRDS. Ch. 4: Cardiovascular Disease. 2013,

2 de Jager DJ, Grootendorst DC, Jager KJ, van Dijk PC, Tomas LM, Ansell D, et al. Cardiovascular and noncardiovascular mortality among patients starting dialysis. JAMA. 2009; 302(16):1782-9.

3 Oh HJ, Lee MJ, Lee HS, Park JT, Han SH, Yoo $\mathrm{TH}$, et al. NT-proBNP: is it a more significant risk factor for mortality than troponin $\mathrm{T}$ in incident hemodialysis patients? Medicine. 2014;93(27):e241.

4 Satyan S, Light RP, Agarwal R. Relationships of $\mathrm{N}$-terminal pro-B-natriuretic peptide and cardiac troponin $\mathrm{T}$ to left ventricular mass and function and mortality in asymptomatic hemodialysis patients. Am J Kidney Dis. 2007; 50:1009-19.

5 Chen T, Hassan HC, Qian P, Vu M, Makris A. High-sensitivity troponin $\mathrm{T}$ and $\mathrm{C}$-reactive protein have different prognostic values in hemo- and peritoneal dialysis populations: a Cohort Study. J Am Heart Assoc. 2018;7(5): 
6 Untersteller K, Girerd N, Duarte K, Rogacev KS, Seiler-Mussler S, Fliser D, et al. NT-proB$\mathrm{NP}$ and echocardiographic parameters for prediction of cardiovascular outcomes in patients with CKD stages G2-G4. Clin J Am Soc Nephrol. 2016;11(11):1978-88.

7 Colbert G, Jain N, de Lemos JA, Hedayati SS. Utility of traditional circulating and imagingbased cardiac biomarkers in patients with predialysis CKD. Clin J Am Soc Nephrol. 2015;10:515-29.

8 Johansen ND, Biering-Sørensen T, Jensen JS, Mogelvang R. Diastolic dysfunction revisited: a new, feasible, and unambiguous echocardiographic classification predicts major cardiovascular events. Am Heart J. 2017;188: $136-46$.

9 Gregg LP, Adams-Huet B, Li X, Colbert G, Jain N, de Lemos JA, et al. Effect modification of chronic kidney disease on the association of circulating and imaging cardiac biomarkers with outcomes. J Am Heart Assoc. 2017; 6(7): 005235

10 Árnadóttir Á, Vestergaard KR, Pallisgaard J, Sölétormos G, Steffensen R, Goetze JP, et al. High-sensitivity cardiac troponin $\mathrm{T}$ is superior to troponin I in the prediction of mortality in patients without acute coronary syndrome. Int J Cardiol. 2018;259:186-91.

11 de Lemos JA, Drazner MH, Omland T, Ayers CR, Khera A, Rohatgi A, et al. Association of troponin $\mathrm{T}$ detected with a highly sensitive assay and cardiac structure and mortality risk in the general population. JAMA. 2010;304(22): 2503-12.

12 Sun L, Ji Y, Wang Y, Ding M, Xie X, Zhu D, et al. High-sensitive cardiac troponin T: a biomarker of left-ventricular diastolic dysfunction in hemodialysis patients. J Nephrol. 2018;31(6):967-73.

13 Sun L, Ji Y, Wang Y, Zhu D, Chen F, Zhang $\mathrm{N}$, et al. Development and internal validation of a prediction model to estimate the probability of left-ventricular diastolic dysfunction in stable maintenance hemodialysis patients without clinical heart failure. Nephron. 2019; 142(4):301-10.
14 Smith K, deFilippi C, Isakova T, Gutiérrez OM, Laliberte K, Seliger S, et al. Fibroblast growth factor 23, high-sensitivity cardiac troponin, and left ventricular hypertrophy in CKD. Am J Kidney Dis. 2013;61(1):67-73.

15 Keller T, Wanner C, Krane V, Kraus D, Genser B, Scharnagl H, et al. Prognostic value of high-sensitivity versus conventional cardiac troponin $\mathrm{T}$ assays among patients with type 2 diabetes mellitus undergoing maintenance hemodialysis. Am J Kidney Dis. 2018;71(6): 822-30.

16 Niizuma S, Iwanaga Y, Washio T, Ashida T, Harasawa S, Miyazaki S, et al. Clinical significance of increased cardiac troponin $\mathrm{T}$ in $\mathrm{pa}$ tients with chronic hemodialysis and cardiovascular disease: comparison to B-type natriuretic peptide and A-type natriuretic peptide increase. Kidney Blood Press Res. 2019;44(5): 1050-62.

17 Bargnoux AS, Morena M, Jaussent I, Maurice F, Chalabi L, Leray-Moragues H, et al. A combined index of cardiac biomarkers as a risk factor for early cardiovascular mortality in hemodialysis patients. Clin Chem Lab Med. 2013;51(9):1865-74

18 Satyan S, Light RP, Agarwal R. Relationships of $\mathrm{N}$-terminal pro-B-natriuretic peptide and cardiac troponin $\mathrm{T}$ to left ventricular mass and function and mortality in asymptomatic hemodialysis patients. Am J Kidney Dis. 2007; 50(6):1009-19.

19 Niizuma S, Iwanaga Y, Yahata T, Miyazaki S Renocardiovascular biomarkers: from the perspective of managing chronic kidney disease and cardiovascular disease. Front Cardiovasc Med. 2017;4:10.

20 Steyerberg EW, Models CP. Statistics for biology and health. New York: Springer; 2009.

21 Moons KG, Altman DG, Reitsma JB, Ioannidis JP, Macaskill P, Steyerberg EW, et al. Transparent reporting of a multivariable prediction model for individual prognosis or diagnosis (TRIPOD): explanation and elaboration. Ann Intern Med. 2015 Jan;162(1):W173.

22 Widera C, Pencina MJ, Bobadilla M, Reimann I, Guba-Quint A, Marquardt I, et al. Incremental prognostic value of biomarkers beyond the GRACE (Global Registry of Acute Coronary Events) score and high-sensitivity cardiac troponin $\mathrm{T}$ in non-ST-elevation acute coronary syndrome. Clin Chem. 2013;59: 1497-505.
23 Cook NR, Ridker PM. Advances in measuring the effect of individual predictors of cardiovascular risk: the role of reclassification measures. Ann Intern Med. 2009;150:795-802.

24 Cook NR, Paynter NP. Performance of reclassification statistics in comparing risk prediction models. Biom J. 2011;53:237-58.

25 Diderholm E, Andrén B, Frostfeldt G, Genberg $\mathrm{M}$, Jernberg $\mathrm{T}$, Lagerqvist $\mathrm{B}$, et al. The prognostic and therapeutic implications of increased troponin T levels and ST depression in unstable coronary artery disease: the FRISC II invasive troponin T electrocardiogram substudy. Am Heart J. 2002;143(5):760-7.

26 Kawel-Boehm N, Kronmal R, Eng J, Folsom A, Burke G, Carr JJ, et al. Left ventricular mass at MRI and long-term risk of cardiovascular events: the multi-ethnic study of atherosclerosis (MESA). Radiology. 2019;293(1):10714.

27 Johansen ND, Biering-Sørensen T, Jensen JS, Mogelvang R. Diastolic dysfunction revisited: a new, feasible, and unambiguous echocardiographic classification predicts major cardiovascular events. Am Heart J. 2017;188: 136-46.

28 Kuznetsova T, Thijs L, Knez J, Herbots L, Zhang Z, Staessen JA, et al. Prognostic value of left ventricular diastolic dysfunction in a general population. J Am Heart Assoc. 2014; 3(3): e000789

29 Han SS, Cho GY, Park YS, Baek SH, Ahn SY, Kim S, et al. Predictive value of echocardiographic parameters for clinical events in patients starting hemodialysis. J Korean Med Sci. 2015;30(1):44-53

30 Iwabuchi $\mathrm{Y}$, Ogawa T, Inoue T, Otsuka K, Nitta K. Elevated E/E' predicts cardiovascular events in hemodialysis patients with preserved systolic function. Intern Med. 2012; 51(2):155-60.

31 Dubin RF, Li Y, He J, Jaar BG, Kallem R, Lash JP, et al. Predictors of high sensitivity cardiac troponin $\mathrm{T}$ in chronic kidney disease patients: a cross-sectional study in the chronic renal insufficiency cohort (CRIC). BMC Nephrol. 2013 Oct 22;14:229. 